14.2;02.4

\title{
Фрагментация молекулы глютамина электронным ударом
}

\author{
() А.В. Папп ${ }^{1}$, В.С. Вукстич ${ }^{1}$, Л.Г. Романова ${ }^{1}$, Т.А. Снегурская ${ }^{2}$, И.Г. Мегела ${ }^{1}$, А.В. Снегурский ${ }^{1, q}$ \\ ${ }^{1}$ Институт электронной физики НАН Украины, Ужгород, Украина \\ ${ }^{2}$ Ужгородский национальный университет, Ужгород, Украина \\ ฯ E-mail: snegursky.alex@gmail.com
}

Поступило в Редакцию 20 июня 2019г.

В окончательной редакции 20 июня 2019 r.

Принято к публикации 5 июля 2019 г.

Методом масс-спектрометрического анализа исследовано образование ионных продуктов однократной и диссоциативной ионизации молекулы глютамина $\left(\mathrm{C}_{5} \mathrm{H}_{10} \mathrm{~N}_{2} \mathrm{O}_{3}\right)$ при ее взаимодействии с электронами малой $(70 \mathrm{eV})$ и высокой $(11.5 \mathrm{MeV})$ энергий. Проведен анализ экспериментальных масс-спектров молекулы при различных дозах облучения $(0,5,10$ и $20 \mathrm{kGy})$, а также припороговых функций выхода ее ионных фрагментов, определены абсолютные значения энергий их появления. Источниками электронных пучков служили трехэлектродная электронная пушка и ускоритель электронов (микротрон). Анализ поведения измеренных масс-спектров молекулы глютамина, облученной при указанных дозах, по сравнению с поведением масс-спектра необлученной молекулы показывает, что высокоэнергетическое облучение исходной молекулы приводит к необратимым изменениям в ее структуре.

Ключевые слова: глютамин, масс-спектр, пучок электронов, микротрон, ионный фрагмент, энергия (потенциал) появления.

DOI: 10.21883/PJTF.2019.20.48394.17942

Ранее в работах [1-3] исследовалось образование ионных фрагментов при взаимодействии электронов больших (порядка $10 \mathrm{MeV}$ ) и малых (до $150 \mathrm{eV}$ ) энергий с молекулами аминокислот триптофана, пролина и валина. Были измерены масс-спектры исследуемых молекул, потенциалы ионизации исходных молекул и потенциалы появления их основных ионных фрагментов. Также были отождествлены основные продукты фрагментации молекул, при этом было найдено, что взаимодействие высокоэнергетических электронов с указанными молекулами не вносит существенных изменений в механизмы их распада, вызывая, тем не менее, стойкие изменения в структуре исходных молекул.

Настоящая работа посвящена масс-спектрометрическому исследованию фрагментации молекулы глютамина $\left(\mathrm{C}_{5} \mathrm{H}_{10} \mathrm{~N}_{2} \mathrm{O}_{3}\right)$ электронами широкого энергетического спектра. Глютамин принадлежит к классу заменимых аминокислот, синтезируемых организмом человека, он широко применяется в качестве пищевых добавок, используемых в современной медицине, в том числе спортивной, обладает способностью ускорять метаболические процессы в мышцах и замедлять катаболические процессы [4]. Поэтому получение информации о поведении данной молекулы под воздействием ионизирующего излучения (тем более высокоэнергетического) представляет значительный интерес как с сугубо научной, так и с практической (в первую очередь относящейся к радиотерапии) точек зрения.

Число работ, посвященных исследованию процессов фрагментации молекулы глютамина под действием электронов, невелико (см., например, [5-7]), а данные о взаимодействии высокоэнергетического излучения с этой молекулой вообще отсутствуют. В указанных работах приводятся масс-спектры исследуемой молекулы, обобщенные в базе данных Национального института стандартов США [8]. В этой базе данных полностью отсутствуют сведения о потенциалах появления фрагментов молекулы глютамина.

В настоящей работе представлены результаты исследования фрагментации молекулы глютамина под воздействием электронного удара с использованием техники пересекающихся молекулярного и электронного пучков и масс-спектрометрического анализа продуктов взаимодействия. Положительно заряженные ионы анализировались по отношению их массы к заряду с помощью модернизированного магнитного массспектрометра МИ-1201 [9]. Методика эксперимента подробно описана нами в предыдущих работах (см., например, [1-3]). Здесь мы только вкратце напомним основные ее моменты. Пучок молекул создавался эффузионным источником с резистивным нагревом рабочего вещества, плотность частиц пучка составляла порядка $10^{10} \mathrm{~mol} / \mathrm{cm}^{3}$. Источником пучка низкоэнергетических $(10-150 \mathrm{eV})$ электронов служила трехэлектродная электронная пушка, энергетический разброс электронов в пучке составлял $\pm 0.1 \mathrm{eV}$, а источником высокоэнергетических электронов служил ускоритель электронов микротрон. Положительные ионы поступали в анализатор масс-спектрометра, разделялись по отношению их массы к заряду $m / z$ и регистрировались системой детектирования полезного сигнала, его циклического накопления во времени и обработки, которая управлялась персональным компьютером. На первом этапе эксперимента проводилось измерение масс-спектра молекулы глютамина в широком диапазоне масс при энергии ионизирующих электронов $70 \mathrm{eV}$ (рис. 1). Далее массспектр отождествлялся, определялись основные ионные 


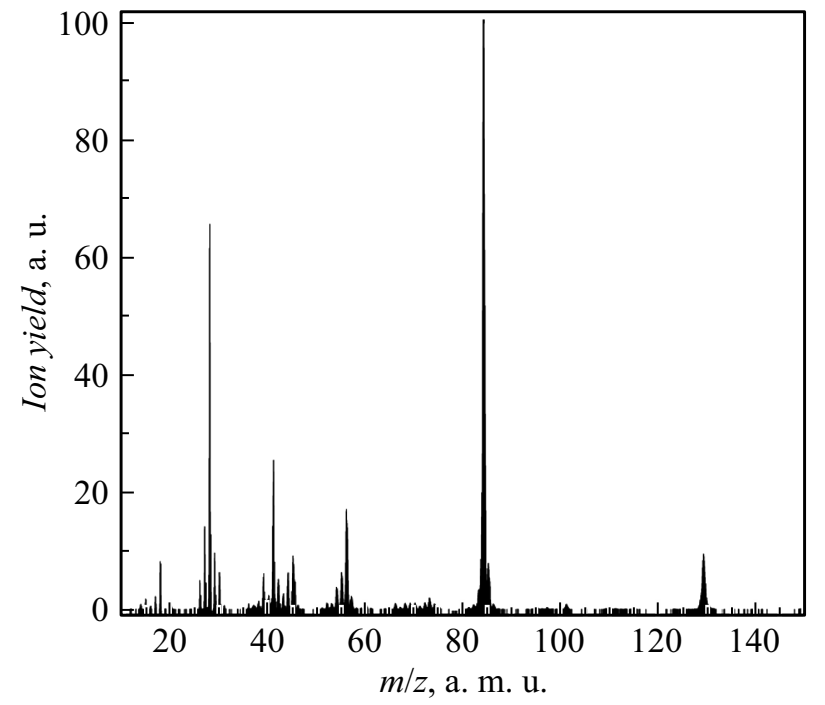

Рис. 1. Масс-спектр необлученной молекулы глютамина.

пики фрагментов глютамина, после чего измерялись зависимости выхода ионов различной массы от энергии электронов вблизи порога процесса. Энергия электронов изменялась автоматически с шагом $0.1 \mathrm{eV}$, а полученные функции диссоциативной ионизации в припороговой области энергий ионизирующих электронов обрабатывались по методу наименьших квадратов, описание которого можно найти в наших предыдущих работах (см., например, [10]). Калибровка энергетической шкалы электронов осуществлялась путем измерения порогов функций ионизации атомов аргона и молекулы азота и их сравнения с известными из литературы данными [10], точность определения энергии электронов составляла $\pm 0.1 \mathrm{eV}$.

На втором этапе работы измерялись масс-спектры этой же молекулы после ее взаимодействия с высокоэнергетическим пучком электронов, который генерировался ускорителем электронов - микротроном М-30. Молекулы глютамина в виде мелкокристаллического порошка облучались электронным пучком с энергией $11.5 \mathrm{MeV}$ при трех дозах облучения: $5 \mathrm{kGy}$ (интегральный поток $1.56 \cdot 10^{13} e / \mathrm{cm}^{2}$ ), $10 \mathrm{kGy}$ (интегральный поток $3.28 \cdot 10^{13} e / \mathrm{cm}^{2}$ ) и $20 \mathrm{kGy}$ (интегральный поток $\left.6.24 \cdot 10^{13} \mathrm{e} / \mathrm{cm}^{2}\right)$. Неоднородность поля облучения в месте нахождения образца не превышала $1 \%$.

Масс-спектр необлученной молекулы глютамина показан на рис. 1. Следует отметить, что он характеризуется значительным количеством ионных линий и в целом подобен приведенному в известной базе данных Национального института стандартов США [8].

Как видно из рисунка, пик, соответствующий образованию материнского иона (массой $m / z=138$ a.m.u.), в измеренном масс-спектре отсутствует, что характерно для большинства аминокислот. Это свидетельствует об эффективном развале иона исходной молекулы непосредственно в ионном источнике масс-спектрометра вследствие его низкой устойчивости к воздействию внешнего возмущения. Доминирующий пик масс-спектра имеет массу $m / z=84$ a.m.u. и обусловлен образованием иона $\mathrm{C}_{4} \mathrm{H}_{8} \mathrm{~N}_{2}^{+}$. Его появление может быть связано с потерей материнской молекулой фрагмента $\mathrm{COOH}$ и атомов $\mathrm{H}, \mathrm{O}$ при разрыве связи $\mathrm{C}=\mathrm{O}$ карбоксильной группы либо (в конкурирующем канале) с отщеплением фрагментов $\mathrm{COOH}, \mathrm{NH}_{2}$ и $\mathrm{H}$.

Другие заметные фрагменты указанного масс-спектра имеют следующие массы (в порядке их убывания): $m / z=56,41$ и 28 a.m.u. Интенсивности соответствующих им пиков спектра в несколько раз уступают интенсивности главного пика при $m / z=84$ a.m.u.

Нами также были измерены начальные участки функций выхода всех четырех перечисленных выше фрагментов необлученной молекулы глютамина. Они представлены на рис. 2. Как видно из рисунка, определенные экспериментально абсолютные величины порогов появления указанных фрагментов составляют $8.5 \pm 0.1 \mathrm{eV} \quad(m / z=84$ a.m.u. $), 13.3 \pm 0.1 \mathrm{eV}$ $(m / z=56$ a.m.u. $), \quad 12.2 \pm 0.1 \mathrm{eV} \quad(m / z=41$ a.m.u. $) \quad$ и $13.9 \pm 0.1 \mathrm{eV} \quad(m / z=28$ a.m.u.). K сожалению, отсутствие подобных сведений в литературе не позволило нам провести сопоставление наших результатов с имеющимися на данный момент.

Пик масс-спектра при $m / z=56$ a.m.u. может быть обусловлен ионами со следующими конфигурациями элементного состава: $\mathrm{C}_{3} \mathrm{OH}_{4}^{+}, \mathrm{C}_{3} \mathrm{NH}_{6}^{+}$и $\mathrm{C}_{2} \mathrm{NOH}_{2}^{+}$. Фрагмент массой $m / z=41$ a.m.u. может соответствовать суммарному вкладу трех положительно заряженных ионов: $\mathrm{C}_{3} \mathrm{H}_{5}^{+}, \mathrm{C}_{2} \mathrm{NH}_{3}^{+}$или $\mathrm{C}_{2} \mathrm{OH}^{+}$. И наконец, выход положительного иона массой $m / z=28$ a.m.u. может быть обусловлен ионами $\mathrm{HC}-\mathrm{NH}\left(\mathrm{CNH}_{2}^{+}\right)$или $\mathrm{CO}^{+}$. Фрагмент с $m / z=28$ a.m.u. характерен для диссоциации всех аминокислот [8] и для большинства из них доминирует в диапазоне массовых чисел 27-30 a.m.u. Фрагмент $\mathrm{CNH}_{2}^{+}$может быть образован с участием как атома азота $\alpha$-аминогруппы, так и атома азота амидной группы молекулы глютамина. Наши предыдущие данные [11] для ряда аминокислот свидетельствуют о том, что этот фрагмент является более структурно устойчивым, чем $\mathrm{CO}$, и формирование азотсодержащего фрагмента имеет бо́льшую вероятность, чем образование фрагмента СО. В случае молекулы глютамина образование ионов с $m / z=56,41$ и 28 a.m.u. также может быть обусловлено вторичной диссоциацией, а именно распадом промежуточного фрагмента массой $m / z=84$ a.m.u.

Облученные на микротроне при указанных выше трех дозах образцы молекул глютамина также исследовались с помощью магнитного масс-спектрометра МИ-1201 по описанной выше методике, а полученные масс-спектры сопоставлялись с исходным масс-спектром необлученного вещества (рис. 3).

Проанализируем масс-спектры, представленные на рис. 3. Относительные интенсивности показанных на рисунке пиков приведены к значению интенсивности доминирующего пика в масс-спектре (при соблюдении одинаковых условий испарения образца в источнике молекул и ионизации в ионном источнике), что позволяет 

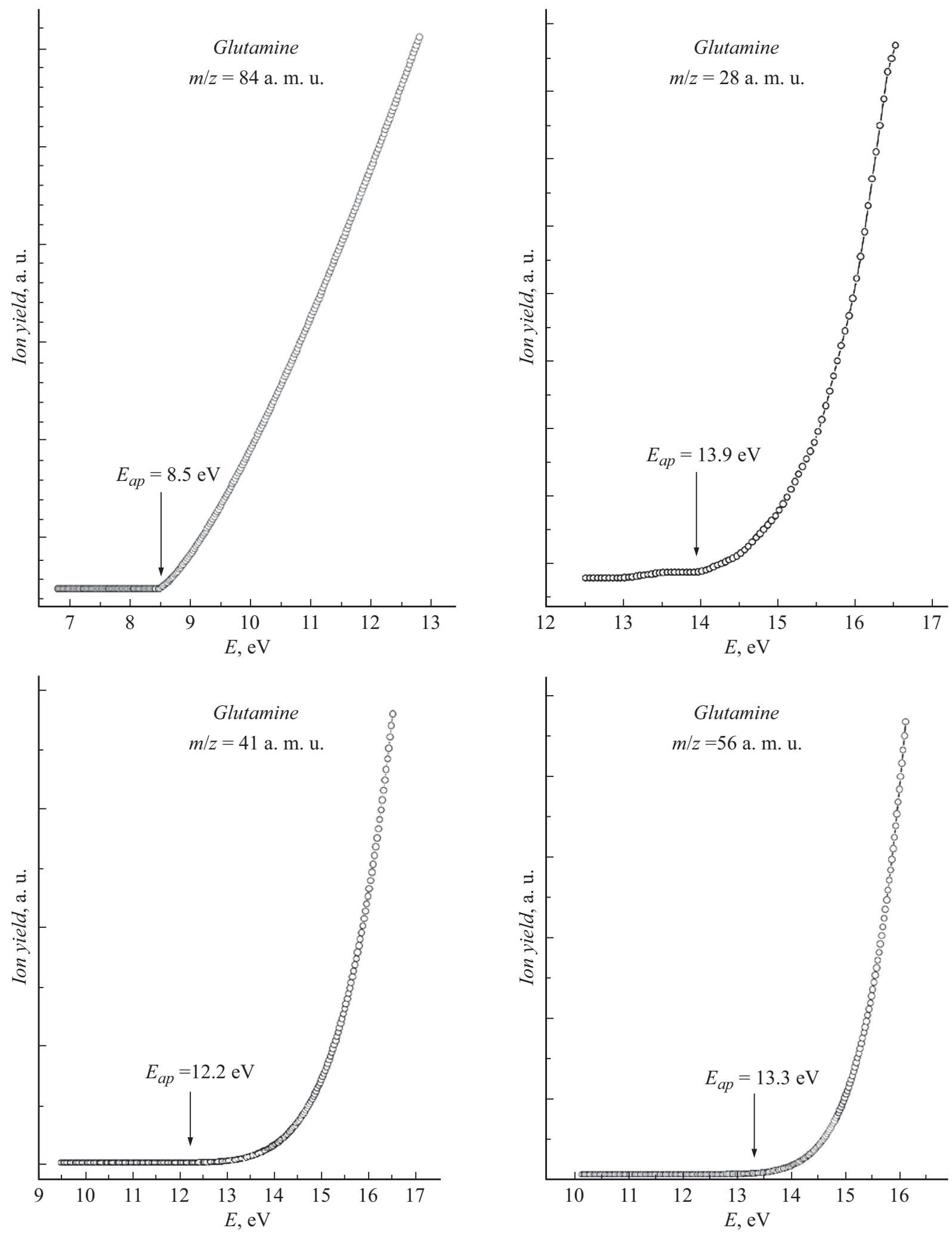

Рис. 2. Начальные участки функций выхода основных ионных фрагментов молекулы глютамина.

провести их сравнение. Масс-спектры образцов, подвергшихся облучению, подобны масс-спектру для необлученной молекулы, однако интенсивности пиков некоторых фрагментов оказываются иными (см. таблицу).
Анализ изменений в масс-спектрах молекулы глютамина вследствие ее облучения высокоэнергетическими электронами дозами 5, 10 и $20 \mathrm{kGy}$ позволяет сделать вывод, что взаимодействие высокоэнергетиче- 


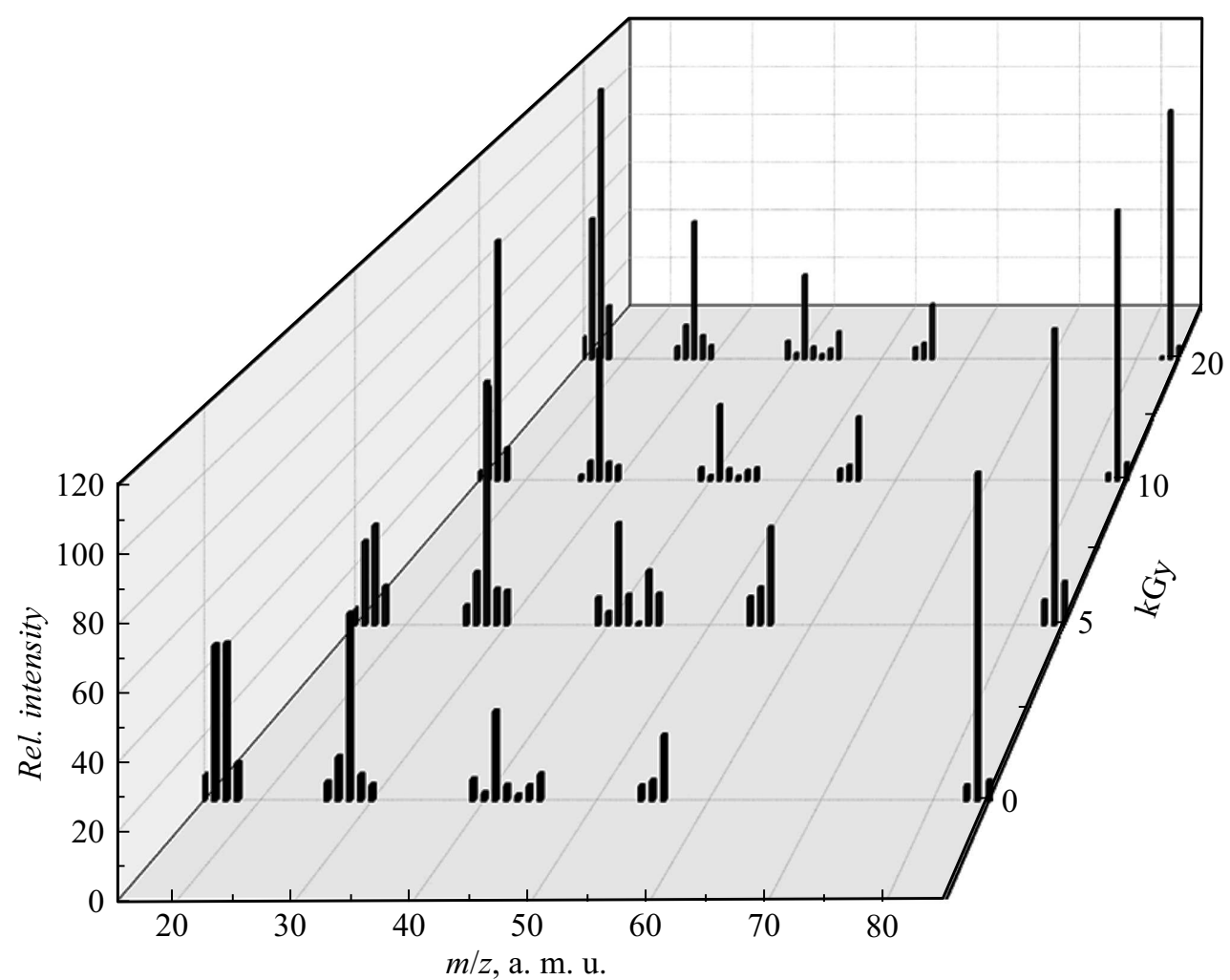

Рис. 3. Масс-спектры необлученной и облученных молекул глютамина. Дозы 5, 10 и $20 \mathrm{kGy}$.

Сравнение интенсивностей основных пиков масс-спектров (приведенных к интенсивности пика массой $m / z=84$ a.m.u.) для необлученной и облученных молекул глютамина

\begin{tabular}{c|c|c|c|c}
\hline \multirow{2}{*}{ Macca, a.m.u } & \multicolumn{4}{|c}{ Доза облучения, kGy } \\
\cline { 2 - 5 } & 0 & 5 & 10 & 20 \\
\hline 28 & 34.1 & 45.1 & 67.8 & 74.7 \\
41 & 30.9 & 34.2 & 39.3 & 41.1 \\
56 & 31.2 & 27.8 & 23.1 & 19.0
\end{tabular}

ских электронов с молекулой глютамина не вызывает появления новых пиков в масс-спектре (рис. 3), однако приводит к заметным изменениям эффективности протекания реакций диссоциативной ионизации молекулы. Таким образом, можно заключить, что нами масс-спектрометрическим методом впервые выполнены исследования фрагментации молекулы глютамина электронным ударом при малых $(70 \mathrm{eV})$ и высоких $(11.5 \mathrm{MeV})$ энергиях. Впервые получены масс-спектры данной молекулы при указанных дозах облучения. Показано, что облучение приводит к разрушению определенной части молекул, причем степень его определяется полученной дозой облучения.

\section{Конфликт интересов}

Авторы заявляют, что у них нет конфликта интересов.

\section{Список литературы}

[1] Вукстич В.С., Романова Л.Г., Мегела И.Г., Папп А.В., Снегурский А.В. // Письма в ЖТФ. 2014. Т. 40. В. 6. С. 64 72.

[2] Вукстич В.С., Романова Л.Г., Мегела И.Г., Папп А.В., Снегурский А.В. // Письма в ЖТФ. 2014. Т. 40. В. 20. C. $39-46$.

[3] Вукстич В.С., Романова Л.Г., Мегела И.Г., Папn А.В., Снегурский А.В. // Письма в ЖТФ. 2017. Т. 43. В. 9. C. 10-19.

[4] Ward J.F. // Advances in radiation biology / Eds J.T. Lett, H. Adler. N.Y.: Academic Press, 1977. P. 181-239.

[5] Zhang Q., Wysocki V.H., Scaraffia P.Y., Wells M.A. // J. Am. Soc. Mass Spectrom. 2005. V. 16. P. 1192-1203.

[6] Wang B., Wu G., Zhou Z., Dai Z., Sun Y., Ji Y., Li W., Wang W., Liu C., Han F., Wu Z. // Amino Acids. 2015. V. 47. P. 2143-2154.

[7] Webb I., Andriole E.J., Poutsma J.C. // Int. J. Mass Spectrom. 2007. V. 267. P. 54-62.

[8] Chemistry Webbook. Standard Reference Database. National Institute of Standards. http://webbook.nist gov

[9] Вукстич В.С., Имре А.И., Снегурский А.В. // ПТЭ. 2011. № 2. C. $66-72$.

[10] Vukstich V.S., Imre A.I., Romanova L.G., Snegursky A.V. // J. Phys. B. 2010. V. 43. P. 185208 (1-6).

[11] Snegursky A.V., Tamuliene J., Romanova L.G., Vukstich V.S Amino acid molecules fragmentation by low-energy electrons. N.Y.: Nova Publ., 2014. 111 p. 